\title{
Global Jitter Motion of the Retinal Image Dynamically Alters the Receptive Field Properties of Retinal Ganglion Cells
}

OPEN ACCESS

Edited by:

Mehdi Adibi,

University of New South Wales,

Australia

Reviewed by:

Masoud Ghodrati,

Monash University, Australia

William Martin Connelly,

University of Tasmania, Australia

${ }^{*}$ Correspondence:

Masao Tachibana

mstchbn@fc.ritsumei.ac.jp

Specialty section:

This article was submitted to

Perception Science,

a section of the journal

Frontiers in Neuroscience

Received: 01 July 2019

Accepted: 30 August 2019

Published: 13 September 2019

Citation:

Matsumoto $A$ and Tachibana $M$ (2019) Global Jitter Motion of the Retinal Image Dynamically Alters the Receptive Field Properties of Retinal Ganglion Cells.

Front. Neurosci. 13:979.

doi: 10.3389/fnins.2019.00979

\author{
Akihiro Matsumoto ${ }^{1,2,3}$ and Masao Tachibana ${ }^{1,4 *}$ \\ 'Department of Psychology, Graduate School of Humanities and Sociology, The University of Tokyo, Tokyo, Japan, \\ ${ }^{2}$ Ritsumeikan Global Innovation Research Organization (R-GIRO), Ritsumeikan University, Kusatsu, Japan, ${ }^{3}$ Danish Research \\ Institute of Translational Neuroscience (DANDRITE), Department of Biomedicine, Aarhus University, Aarhus, Denmark, \\ ${ }^{4}$ Research Organization of Science and Technology, Ritsumeikan University, Kusatsu, Japan
}

Fixational eye movements induce aperiodic motion of the retinal image. However, it is not yet fully understood how fixational eye movements affect retinal information processing. Here we show that global jitter motion, simulating the image motion during fixation, alters the spatiotemporal receptive field properties of retinal ganglion cells. Using multi-electrode and whole-cell recording techniques, we investigated lightevoked responses from ganglion cells in the isolated goldfish retina. Ganglion cells were classified into six groups based on the filtering property of light stimulus, the membrane properties, and the cell morphology. The spatiotemporal receptive field profiles of retinal ganglion cells were estimated by the reverse correlation method, where the dense noise stimulus was applied on the dark or random-dot background. We found that the jitter motion of the random-dot background elongated the receptive filed along the rostral-caudal axis and temporally sensitized in a specific group of ganglion cells: Fast-transient ganglion cells. At the newly emerged regions of the receptive field local light stimulation evoked excitatory postsynaptic currents with large amplitude and fast kinetics without changing the properties of inhibitory postsynaptic currents. Pharmacological experiments suggested two presynaptic mechanisms underlying the receptive field alteration: (i) electrical coupling between bipolar cells, which expands the receptive field in all directions; (ii) GABAergic presynaptic inhibition from amacrine cells, which reduces the dorsal and ventral regions of the expanded receptive field, resulting in elongation along the rostral-caudal axis. Our study demonstrates that the receptive field of Fast-transient ganglion cells is not static but dynamically altered depending on the visual inputs. The receptive field elongation during fixational eye movements may contribute to prompt firing to a target in the succeeding saccade.

Keywords: retina, retinal ganglion cells, receptive field, eye movements, gap junctions

\section{INTRODUCTION}

In living animals, the retina receives unstable visual inputs induced by movements of body, head, and eyes (Land, 2009). Even when an animal is fixating an object, the whole image on the retina is shifted by the presence of incessant microscopic eye movements ("fixational eye movements") (Martinez-Conde et al., 2004; Rucci and Poletti, 2015). Once fixational eye movements are 
stabilized, visual perception fades rapidly (Yarbus, 1967; Murakami, 2006). It has been shown that fixational eye movements affect visual performance such as visual acuity (Keesey, 1960), contrast sensitivity (Tulunay-Keesey and Jones, 1976), and detection of visual features (Rucci et al., 2007). Physiological evidence indicates that the image motion induced by fixational eye movements prevents the adaptation of neural activity (Martinez-Conde et al., 2004; Rucci and Poletti, 2015). Involuntary small eye movements during fixation, called microsaccades, increase firing activity of primate visual cortical neurons (V1, Martinez-Conde et al., 2000; area MT, Bair and O'Keefe, 1998). Retinal ganglion cells (GCs) respond with higher firing rate to the jittering image than the static image, together with synchronization (Greschner et al., 2002) or with decorrelation (Segal et al., 2015).

In natural vision, animals repeat fixations and brief gaze shifts (saccades) (Wurtz, 2008; Land, 2009). It has been shown that firing of GCs is increased (Noda and Adey, 1974) or suppressed (Roska and Werblin, 2003; Tien et al., 2015) by saccade-like image shift depending on cell types. A burst firing is evoked by image recurrence across eye-movement-like image transitions in mouse specific GCs (Krishnamoorthy et al., 2017). Glycinergic and GABAergic inhibitory inputs from amacrine cells seem to contribute to the firing modulation during the rapid image shift (Roska and Werblin, 2003; Tien et al., 2015; Krishnamoorthy et al., 2017).

In our previous study, applying a multi-electrode array to GCs in the goldfish isolated retina, we showed that firing properties of specific GC groups were modulated by a rapid shift of a target following a period of jitter motion of a global random-dot background (Matsumoto and Tachibana, 2017). In particular, the response latency to a rapidly moving target was shortened in Fast-transient (Ft) GCs only when rapid motion was preceded by global jitter motion. Intriguingly, the response modulation was specific to rapid motion along the rostral-caudal axis. These results suggest that the receptive field (RF) properties of Ft GCs may have been altered during a period of global jitter motion prior to rapid motion. However, global jitter motion per se did not evoke firing in Ft GCs, and thus, it remains to be solved how global jitter motion alters the RF properties of Ft GCs and what mechanisms underlie the alteration.

Here, applying the whole-cell clamp technique as well as the multi-electrode technique to goldfish retinal GCs, we analyzed the effects of global jitter motion on the spatiotemporal RF profiles. We found that the RF of $\mathrm{Ft}$ GCs was spatially elongated along the rostral-caudal axis and temporally sensitized by jitter motion. At the newly emerged regions, local light stimulation frequently evoked excitatory postsynaptic currents with large amplitude and fast kinetics. Pharmacological experiments suggested that the RF alterations were mediated by activation of electrical coupling between bipolar cells and GABAergic inhibition from amacrine cells to bipolar cell terminals. Elongation of the RF of Ft GCs during jitter motion may contribute to prompt response to a rapidly moving target in the succeeding saccade.

\section{MATERIALS AND METHODS}

\section{Experimental Model and Subject Details}

Goldfish (Carassius auratus; $8-12 \mathrm{~cm} ; n=62$ ) was used for the experiments. Animals were kept in a room maintained at $23^{\circ} \mathrm{C}$ on a $12 \mathrm{~h}$ light/dark cycle. All protocols complied with "A Manual for the Conduct of Animal Experiments in The University of Tokyo" and "Guiding Principles for the Care and Use of Animals in the Field of Physiological Sciences, The Physiological Society of Japan."

\section{Method Details}

\section{Retinal Preparation}

Goldfish were dark-adapted for more than $1 \mathrm{~h}$ before experiments. Under a dim red light, a goldfish was doublepithed, and eyes were enucleated. The following procedure was performed under a stereomicroscope equipped with infrared (IR) image converter (C5100, Hamamatsu photonics) and IR illuminator (HVL-IRM, Sony). After the cornea and lens were ablated, the eye cup was treated with a mixture of hyaluronidase and collagenase ( $4 \mathrm{mg} / \mathrm{mL}$ each, Sigma-Aldrich Corp.) for a few min. A small cut was made at the dorsal part of the eye cup as a landmark and thus the ventral retina isolated from the pigment epithelium was properly oriented and positioned on the multi-electrode array or in the recording chamber for whole-cell recordings.

\section{Recordings}

For multi-electrode recordings (Figures 1, 2), the isolated retina was placed on the electrode array (60 electrodes, electrode diameter $30 \mu \mathrm{m}$, electrode spacing $200 \mu \mathrm{m}$; 60pMEA200/30iRTi, Multichannel Systems) with the GC layer facing down, and light stimulation was applied from the photoreceptor side. The recorded signals were stored at $16 \mathrm{kHz}$ through $\mathrm{AD}$ converter with 16 channels (PowerLab 16/35; AD Instruments). The retina was continuously superfused with an extracellular solution bubbled with $95 \% \mathrm{O}_{2} / 5 \% \mathrm{CO}_{2}$ at the rate of $1 \mathrm{~mL} / \mathrm{min}$. The solution consisted of (in $\mathrm{mM}$ ) $106 \mathrm{NaCl}, 2.6 \mathrm{KCl}, 28$ $\mathrm{NaHCO}_{3}, 2.5 \mathrm{CaCl}_{2}, 1 \mathrm{MgCl}_{2}, 1 \mathrm{Na}$-pyruvate, $10 \mathrm{D}$-glucose, $4 \mathrm{mg} / \mathrm{L}$ phenol red. For pharmacological experiments, drugs were added to the extracellular solution. Drugs were obtained from Sigma-Aldrich. Recorded spike discharges were bandpass filtered between 100 and $3,000 \mathrm{~Hz}$ and sorted into single unit activities by principal component analysis (PCA) and the template-matching method with custom programs using MATLAB (Lewicki, 1998; Zhang et al., 2004; Matsumoto and Tachibana, 2017). For further analysis, we selected up to 3 single units/electrode, showing robust light-evoked responses based on two criteria: responsibility and reliability. Responsibility was evaluated by comparing mean firing probability before and during light stimulus using two-tailed $t$-test. Reliability was evaluated by trial-to-trial variability in spike counts during light stimulus based on Pearson's correlation. To verify the accuracy of sorting, we calculated the auto-correlation of the sorted spike train for each unit, and confirmed a lack of events corresponding to the refractory period of spikes (Supplementary Figure S1A) (Lewicki, 1998; Rey et al., 2015). 


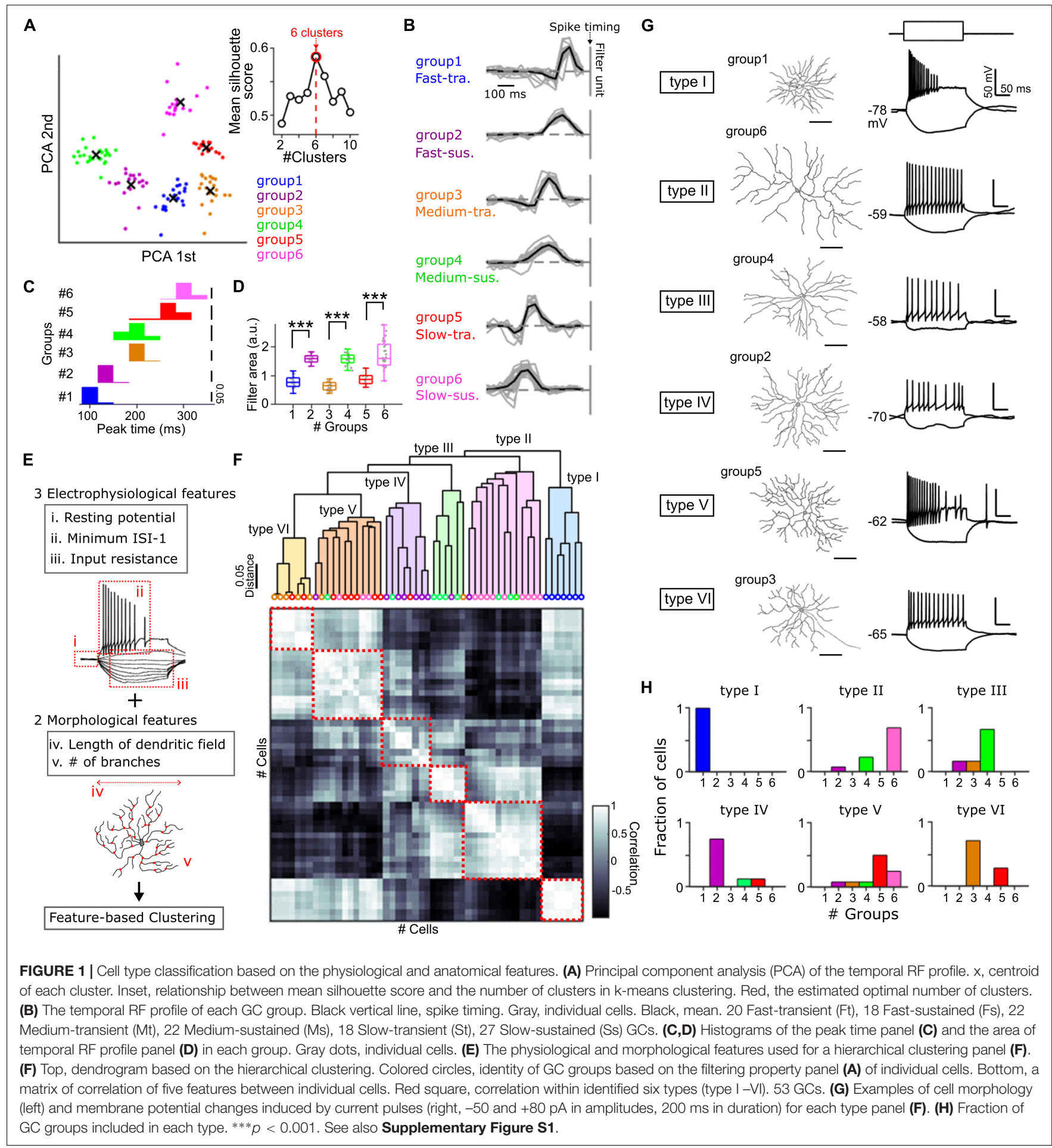

For whole-cell recordings (Figures 1, 3, 4), the isolated retina was placed on the recording chamber with the GC layer facing up, and light stimulation was applied from the photoreceptor side. The retina was continuously superfused with the bubbled extracellular solution. The pipette was filled with intracellular solution (in $\mathrm{mM}$ ): $128 \mathrm{~K}$ gluconate, $10 \mathrm{KCl}, 10 \mathrm{Hepes,} 0.5 \mathrm{EGTA}$,
$0.05 \mathrm{CaCl}_{2}, 2 \mathrm{MgCl}_{2}, 5$ ATP-Na, 0.5 GTP-Na 3 , and $0.08 \%$ Lucifer yellow- $2 \mathrm{~K}$ ( $\mathrm{pH} 7.4$ with $\mathrm{KOH}$ ) for current-clamp recordings, and $118 \mathrm{CsMeSO}_{3}, 10$ TEA-Cl, 10 Hepes, 0.5 EGTA, $0.05 \mathrm{CaCl}_{2}, 2$ $\mathrm{MgCl}_{2}, 5$ ATP-Na $2,0.5$ GTP-Na 3 , 5 QX314-Br, and 0.08\% Lucifer yellow- $2 \mathrm{~K}$ ( $\mathrm{pH} 7.4$ with $\mathrm{CsOH}$ ) for voltage-clamp recordings. $\mathrm{E}_{\mathrm{Cl}}$ was calculated as $-55 \mathrm{mV}$. The membrane potential was 


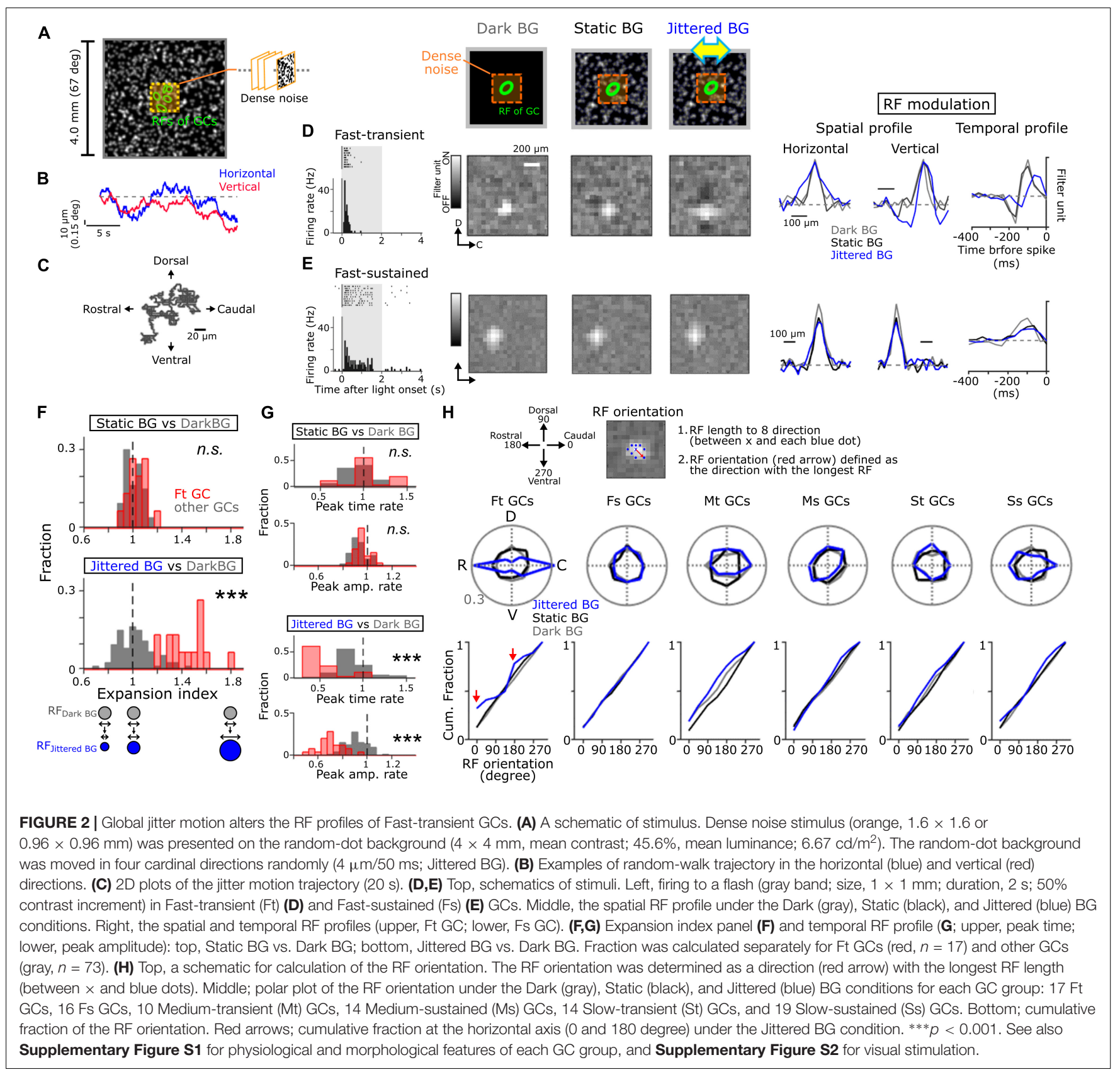

corrected for liquid junction potential which was measured before experiments. For pharmacological experiments (Figure 5), we added picrotoxin (GABA receptor blocker; $100 \mu \mathrm{M}$, Sigma) or mefloquine (gap junction blocker; $10 \mu \mathrm{M}$, Sigma) to the extracellular solution. Recordings were performed using EPC 10 (HEKA Electronik) controlled by Pactchmaster (version 2.73.5). Current and voltage records were sampled at $16 \mathrm{kHz}$ and lowpass filtered at $2.9 \mathrm{KHz}$. We used a borosilicate glass electrode (CNC 1.5; Ken Enterprise), which was pulled by a puller (P97; Sutter Instrument). The resistance of recording pipettes was 6$11 \mathrm{M} \Omega$. For cell group identification, light-evoked spikes were recorded in the cell-attached mode before whole-cell recording.

\section{Light Stimulation}

Light patterns were generated by Psychtoolbox3 on MATLAB (Mathworks) (Brainard, 1997; Pelli, 1997). For multi-electrode recordings, a multi-electrode array was placed on the stage of an inverted microscope (IX70; Olympus). The light stimulus was projected from a cathode-ray tube display (S501J, refresh rate $60 \mathrm{~Hz}, 1,280 \times 1,024$ pixels, Iiyama) to the photoreceptor layer of the retina through optics. For whole-cell recordings, a patch pipette was approached from the ganglion cell side. The light stimulus was projected from a DLP projector (L51W, refresh rate $60 \mathrm{~Hz}, 1,280 \times 1,024$ pixels, NEC) to the photoreceptor layer through an objective 


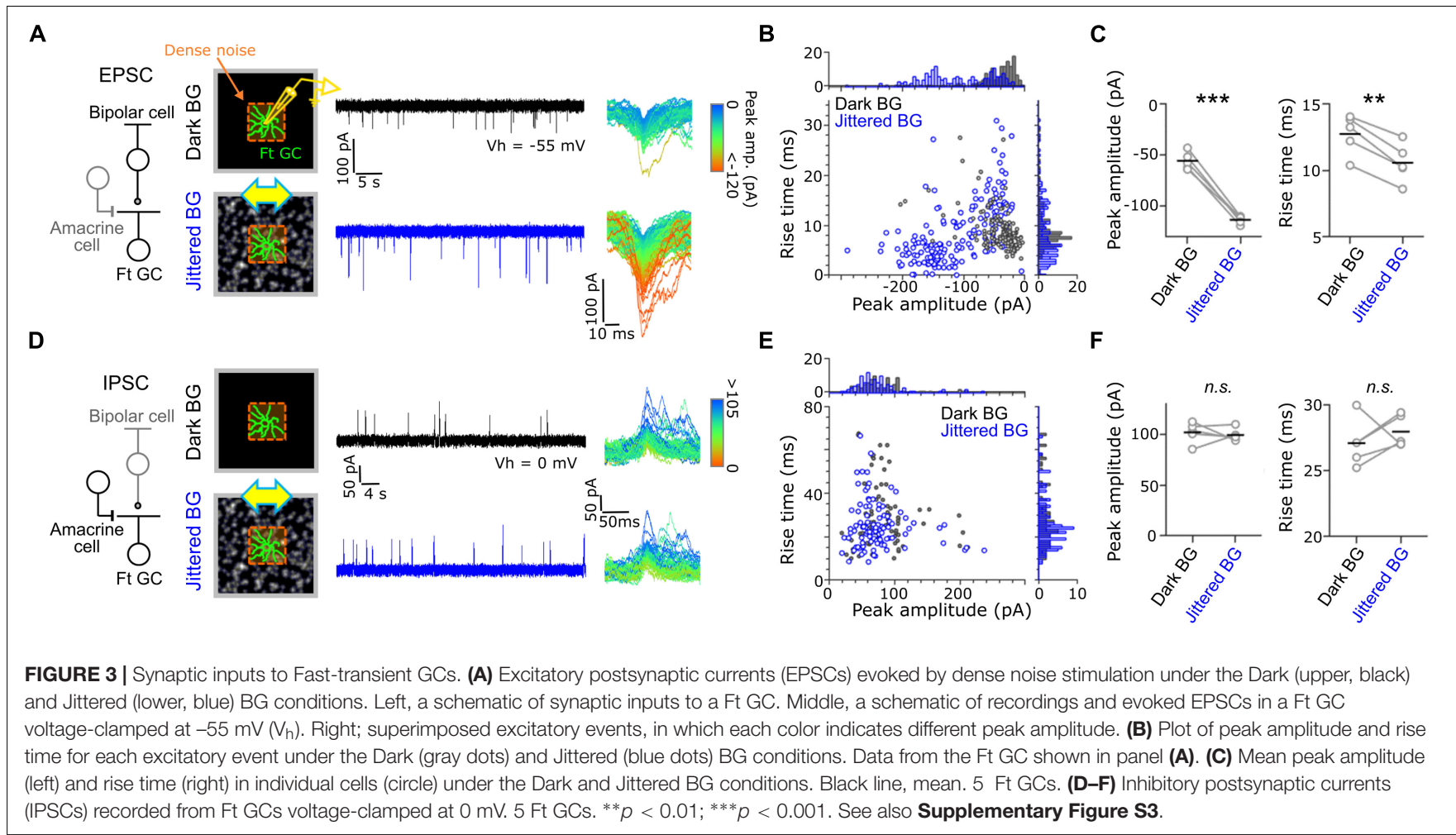

lens $(4 \times 10.10$, Nikon), with which a condenser lens of an upright microscope (Eclipse E600-FN; Nikon) was replaced. In either recording condition, the stimulus image was not distorted because the electrode was positioned on the opposite side of the incident light.

We used a background frame $(4.0 \times 4.0 \mathrm{~mm})$ which was either uniformly dark or a Gaussian-filtered $(\sigma, 40 \mu \mathrm{m})$ randomdot pattern $\left(51,325\right.$ dots $/ 1,000^{2}$ pixels, $4 \mu \mathrm{m} /$ pixel, Figure 1A, see details in Matsumoto and Tachibana, 2017). Background Contrast $\left(C_{\mathrm{BK}}\right)$ was calculated by

$$
C_{\mathrm{BK}}=\left(\text { luminance }_{\mathrm{BK}}-\text { luminance }_{\mathrm{dark}}\right) / \text { luminance }_{\max }
$$

where luminance $_{\mathrm{BK}}$, luminance dark, $_{\text {, and luminance }}$ max were the mean intensity of the random-dot pattern, 0.11 and $14.4 \mathrm{~cd} / \mathrm{m}^{2}$, respectively. We introduced global jitter motion to simulate the in vivo fixational eye movements of goldfish (Figures 2A-C) (Easter et al., 1974; Mensh et al., 2004). Global jitter motion was a horizontally biased random walk, in which a shift $(4 \mu \mathrm{m} / 50 \mathrm{~ms})$ to one of the four cardinal direction occurred in each stimulus frame, and the probability of horizontal shift was two times higher than that of vertical shift.

\section{Receptive Field Estimation}

The spatiotemporal receptive field (RF) was estimated by the reverse correlation method (Meister et al., 1994; Matsumoto and Tachibana, 2017). The retina was stimulated with dense noise consisted of pseudorandom (M-sequence) checkerboard patterns. Each frame $(32 \times 32$ pixels with black or white; pixel size, $50 \times 50 \mu \mathrm{m}$ or $30 \times 30 \mu \mathrm{m})$ was updated at $30 \mathrm{~Hz}$. The dense noise was placed on the center region $(1.6 \times 1.6$ or $0.96 \times 0.96 \mathrm{~mm}$; Figure $\mathbf{2 A}$, orange) of the background $(4.0 \times 4.0 \mathrm{~mm})$. The checkerboard frames that preceded each spike discharge were averaged (STA, spike-triggered average).

To define the orientation and size of the RF, we determined an "edge" (a position of a pixel with the intensity six times higher than the $\mathrm{SD}$ of the intensity in uncorrelated image) along 8 directions $\left(0-315^{\circ}, \Delta 45^{\circ}\right)$ from the RF "center" (a pixel with maximal intensity). Eight edges were fitted by an ellipse based on the method of least squares. RF size was calculated by a length of major axis in the fitted ellipse. RF orientation (Figure $\mathbf{2} \mathbf{H}$ ) was defined by the direction with the longest length among eight directions. The temporal RF profile was obtained by calculating the mean intensity of $3 \times 3$ pixels in the RF center region for a series of the averaged frames.

The temporal RF profile was used for classification of GC groups (Matsumoto and Tachibana, 2017; Figures 1A-D and Supplementary Figure S1B). In brief, we first created an input matrix from the temporal RF profile of each GC, in which the temporal RF profiles were upsampled using interp function with a rate of 3 in MATLAB, and smoothed by a moving average filter. After the smoothing, the temporal filters were downsampled to the original rate using downsample function in MATLAB. Next, principal component analysis (PCA) was applied to visualize the features of temporal RFs (Figure 1A). Then, clustering was performed by $\mathrm{k}$-means clustering using kmeans function in MATLAB. The number of 


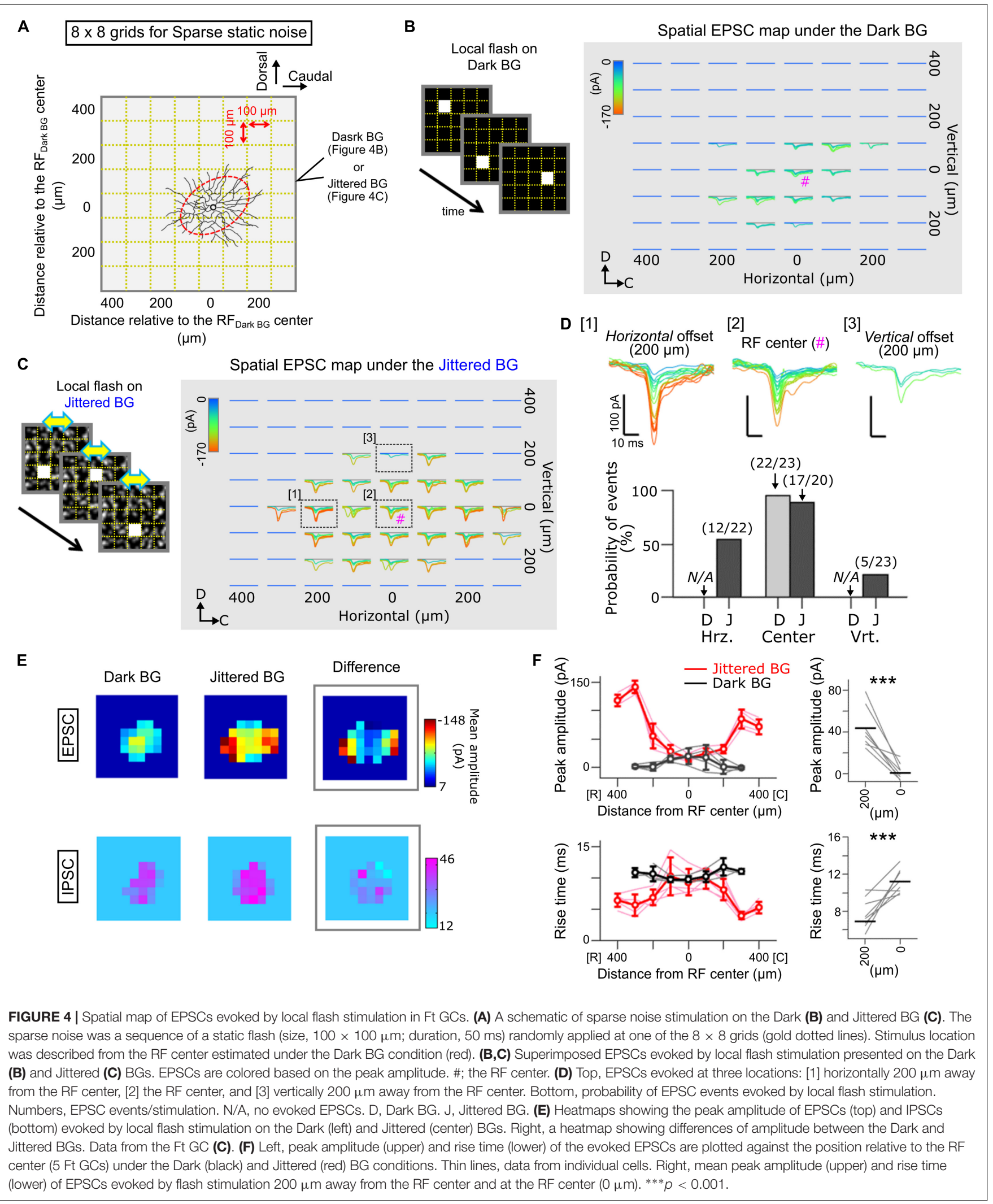




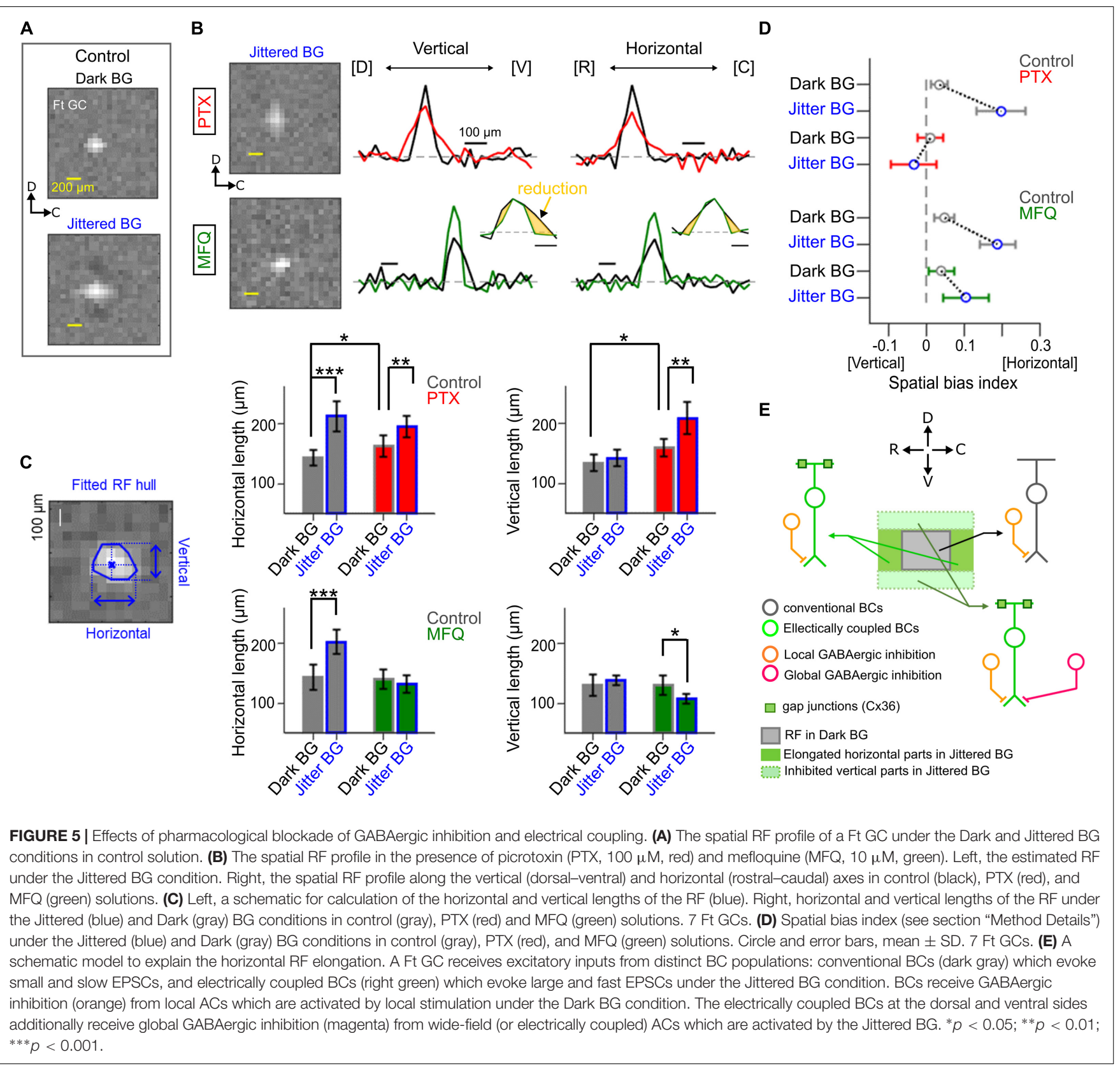

clusters was determined based on the mean silhouette scores $[\mathrm{s}(i)]$ (Figure 1A),

$$
s(i)=b(i)-a(i) / \max \{a(i), b(i)\},
$$

where $i$ is each cell, a $(i)$ is average distance between $i$ and all other points included in the same cluster, and $\mathrm{b}(i)$ is the smallest average distance of $i$ to all other points (Rousseeuw, 1987; Yuan and Yang, 2019). We adopted the cluster numbers with the highest mean silhouette score for k-means clustering (Martínez et al., 2017).

To quantify the horizontal and vertical lengths of RF, we first fitted a hull to the RF (Figure 5C) using convex hull function in MATLAB. A centroid where the horizontal and vertical axes crossed was calculated in the fitted hull, and then, we measured the horizontal and vertical lengths through the centroid. To quantify the spatial bias of the RF profile, the spatial bias index (SBI) was defined by,

$$
\begin{array}{r}
S B I=\left(\text { length }_{\text {Horizontal }}-\text { length }_{\text {Vertical }}\right) / \\
\left(\text { length }_{\text {Horizontal }}+\text { length }_{\text {Vertical }}\right)
\end{array}
$$

where length ${ }_{\text {Horizontal }}$ and length $h_{\text {Vertical }}$ are the measured $\mathrm{RF}$ length along the horizontal and vertical axes, respectively 
(Figure 5D). The positive and negative values indicate the horizontal and vertical bias of the RF, respectively.

\section{Waveform Analysis}

For whole-cell voltage-clamp recordings (Figures 3, 4), wave event was detected as the postsynaptic current when the current amplitude crossed a threshold defined by

$$
\text { Threshold }=\mid \text { Mean }_{\mathrm{c}} \mid+\mathrm{SD}_{\mathrm{c}} * 3,
$$

where $\mathrm{C}$ indicates the currents recorded during dense noise stimulation. To quantify the postsynaptic currents, we calculated the amplitude and rise time of each detected wave event. The rise time was defined as the time required for the response to reach from $63 \%$ to $100 \%$ of the peak.

\section{Analysis of Membrane Properties}

To characterize the membrane properties of GCs, we recorded the membrane potential changes induced by current pulses (duration, $200 \mathrm{~ms}$; amplitude, from -50 to $100 \mathrm{pA}$; increment, $10 \mathrm{pA}$ ). For hierarchical clustering (Figure 1F), we used three features (Supplementary Figure S1D): the resting membrane potential, the maximum instantaneous firing rate, and the input resistance. The resting membrane potential was calculated as an average potential $100 \mathrm{~ms}$ before application of current pulses. The maximum firing rate was calculated as an inverse of minimum inter-spike intervals during current pulses [Supplementary Figure S1D, (i)]. The input resistance was calculated as a slope of the current-voltage function below subthreshold membrane potentials [Supplementary Figure S1D, (ii)]. We performed a hierarchical clustering based on the correlation distance in the standardized feature space using linkage and dendrogram functions in MATLAB (Baden et al., 2016).

\section{Morphological Analysis}

Lucifer yellow was introduced through a recording patch pipette to visualize the cell morphology. Using Image J (NIH) and customized program in MATLAB, we quantified the dendritic field, shape, and dendritic branches of each GC (Figure 1 and Supplementary Figure S1E). The dendritic tips of a stained GC were fitted to a polygon, and then its major axis and the aspect ratio of major to minor axis were used as the measure of the length and the shape, respectively. The number of branches normalized by an area of the polygon was used as the measure of the dendritic branches.

\section{Quantification and Statistical Analysis}

In Figures 2F-H, Kolmogorov-Smirnov test (KS test) was used. In Figure $\mathbf{2 H}$, Hodges-Ajne test was used to evaluate the bias in distribution of the RF orientation. In Figures 3C,F, 4F, paired $t$-test was used. In Figure 5C, Mann-Whitney $U$ test (MWU test) with Tukey's post hoc test was used. All measures for population data were described as mean \pm SD. Error bar indicates SD. ${ }^{* * *} p<0.001$; ${ }^{* *} p<0.01{ }^{*} p<0.05$.

\section{RESULTS}

\section{Classification of Retinal Ganglion Cells Based on the Physiological and Anatomical Features}

Applying a multi-electrode array to the goldfish isolated retina, we estimated the spatiotemporal receptive field (RF) profiles of retinal ganglion cells (GCs) by the reverse correlation method (Meister et al., 1994). The shape of the temporal RF is one of the criteria to define ganglion cell types functionally (DeVries and Baylor, 1997; Hilgen et al., 2017; Matsumoto and Tachibana, 2017). To dissect GC types, we performed feature detection using principal component analysis for the temporal RF (Matsumoto and Tachibana, 2017), and the resulting features were clustered into six GC groups based on k-means clustering (Figures 1A,B and Supplementary Figure S1B; Rousseeuw, 1987; Yuan and Yang, 2019). The differences among the GC groups were summarized by two temporal features: peak time (Fast/Medium/Slow; Figure 1C) and kinetics (transient/sustained; Figure 1D). Thus, individual GC groups were defined as Fast-transient (Ft, group 1), Fast-sustained (Fs, group 2), Medium-transient (Mt, group 3), Medium-sustained (Ms, group 4), Slow-transient (St, group 5), and Slow-sustained (Ss, group 6) GCs (Figure 1B and Supplementary Figure S1C).

We then assessed whether each GC group could share common physiological and anatomical features. We performed whole-cell recordings from randomly selected GCs and dye loading through the recording pipette to visualize the cell morphology (Supplementary Figures S1D-F). Based on three physiological features which represent the membrane properties (Figure 1E, i-iii) and two morphological features (Figure 1E, iv, v), a hierarchical clustering (Martínez et al., 2017; Gouwens et al., 2019) revealed that GCs were branched into six types (Figure 1F, type I to VI). Intriguingly, each type defined by physiological and anatomical features was populated dominantly by a GC group defined by the filtering property (Figures $\mathbf{1 G}, \mathbf{H}$ ). These results highlight the correlation between the filtering property and the physiological and anatomical features to characterize GCs.

\section{Global Jitter Motion Changes Spatiotemporal Receptive Field Profiles of a Specific Group of Retinal Ganglion Cells}

To explore how the RF properties of GCs are affected by fixational eye movements, dense noise stimulus was presented on a large background (BG; $4 \times 4 \mathrm{~mm}$ on the retina, visual angle $>\sim 67^{\circ}$; Figure 2A; Macy and Easter, 1981). We used three kinds of BG patterns: a uniformly dark pattern ("Dark BG"), a static randomdot pattern ("Static BG," a Gaussian-filtered random-dot pattern; mean luminance, $6.67 \mathrm{~cd} / \mathrm{m}^{2}$ ), and a jittered random-dot pattern ("Jittered BG," the Gaussian-filtered random-dot pattern jittered randomly) (Matsumoto and Tachibana, 2017). The jitter motion was a horizontally biased random walk (probability of horizontal shift/vertical shift $=2$; each shift, $4 \mu \mathrm{m} / 50 \mathrm{~ms}$; Figures $2 B, C$ and Supplementary Figure S2C) that simulated the goldfish 
fixational eye movements (Easter et al., 1974; Mensh et al., 2004). Both the Static and Jittered BGs covered the area far away from the RFs of recorded GCs (Supplementary Figure S2A). Based on the responses to dense noise stimulus, we estimated the spatiotemporal RF of GCs under different BG conditions: $\mathrm{RF}_{\text {DarkBG }}$ (gray), $\mathrm{RF}_{\text {StaticBG }}$ (black), and $\mathrm{RF}_{\text {Jittered } \mathrm{BG}}$ (blue).

We found that the Jittered $\mathrm{BG}$ altered the spatiotemporal RF profile of Ft GCs (Figure 2D). The size of $\mathrm{RF}_{\text {JitteredBG }}$ (major axis of the RF; $161.9 \pm 13.2 \mu \mathrm{m}, 21 \mathrm{Ft}$ GCs) was larger than that of either $\mathrm{RF}_{\mathrm{DarkBG}}(112.5 \pm 13.7 \mu \mathrm{m}, p=0.001$; paired $t$-test) or $\mathrm{RF}_{\text {StaticBG }}(115.6 \pm 22.9 \mu \mathrm{m}, p=0.002)$. Expansion index, the ratio of the RF size under different $B G$ conditions, was large only under the Jittered BG condition (Jittered BG vs. Dark BG, $p=8.91 \times 10^{-9}$; Static BG vs. Dark BG, $p=0.38$; KS test, $21 \mathrm{Ft}$ GCs; Figure 2F, red). Other GC groups did not show prominent changes (Figures 2E,F, gray).

The temporal profile of $\mathrm{RF}_{\text {JitteredBG }}$ in Ft GCs showed faster peak time and smaller peak amplitude than those of $\mathrm{RF}_{\text {DarkBG }}$ and $\mathrm{RF}_{\text {StaticBG }}$ (Figures 2D,G), indicating that the Jittered BG sensitized firing and input integration kinetics. These alterations of the temporal RF profile were specific to Ft GCs (peak time, Jittered BG vs. Dark BG, $p=2.35 \times 10^{-9}$, Static BG vs. Dark BG, $p=0.097$; peak amplitude, Jittered BG vs. Dark BG, $p=9.03 \times 10^{-9}$, Static BG vs. Dark BG, $p=0.084 ; \mathrm{KS}$ test, $21 \mathrm{Ft}$ GCs; Figure 2G, red). The Jittered BG did not affect the temporal RF profile of other GC groups (Figures 2E,G, gray), indicating that the RF alterations are not ascribed to light adaptation to the random-dot background.

Intriguingly, $\mathrm{RF}_{\text {JitteredBG }}$ of Ft GCs was oriented along the retinal rostral-caudal (horizontal) axis ( $p=0.019$, HodgesAjne test, 21 Ft GCs; Figure 2H, blue), indicating that the RF expansion is biased along the retinal horizontal axis but not along the dorsal-ventral (vertical) axis (Figure 2H, bottom, red arrows). Since $\mathrm{RF}_{\text {DarkBG }}$ and $\mathrm{RF}_{\text {StaticBG }}$ did not show orientation bias $\left(\mathrm{RF}_{\mathrm{DarkBG}}, p=0.7763\right.$; $\mathrm{RF}_{\text {StaticBG }}, p=0.7763$; Hodges-Ajne test, 21 Ft GCs), the horizontal bias is not intrinsic to Ft GCs. Other GC groups showed no significant orientation bias under three BG conditions. It should be noted that the non-coherent (flickering) random noise BG did not alter the spatial RF profile of Ft GCs (RF size, Dark BG, $125.6 \pm 21.1 \mu \mathrm{m}$; Flickering random noise BG, $121.9 \pm 17.6 \mu \mathrm{m}, p=0.31$; paired $t$-test, $17 \mathrm{Ft}$ GCs; Supplementary Figure S2B). Therefore, global jitter motion is essential for the RF alterations in Ft GCs.

\section{Synaptic Currents Evoked by the Dense Noise Stimulus Under the Jittered BG Condition in Ft GCs}

Effects of the global jitter motion on the spatiotemporal RF profiles were prominent in Ft GCs. To elucidate the underlying mechanisms, we recorded postsynaptic currents from Ft GCs in the whole-cell voltage-clamp configuration (Figure 3). Ft GCs were identified based on the temporal RF profile estimated by firing to dense noise stimulus in the cell-attached configuration. We found that excitatory postsynaptic currents (EPSCs) under the Jittered BG condition were larger in amplitude (Jittered BG, $110.2 \pm 56.8$ pA; Dark BG, $-42.8 \pm 33.9$ pA; $p=0.001$, paired t-test, 5 Ft GCs), and faster in kinetics (Jittered BG, $-110.2 \pm 56.8$ pA; Dark BG, $-42.8 \pm 33.9 \mathrm{pA} ; p=3.85 \times 10^{-5}$, paired $t$-test, 5 Ft GCs) than those under the Dark BG condition (Figures 3B,C). EPSCs under the Static BG condition were not significantly different in both amplitude and kinetics from those under the Dark BG condition (Static BG, $-45.7 \pm 20.9$ pA, $10.6 \pm 2.5 \mathrm{~ms}$, ps $>0.3$; paired $t$-test, 5 Ft GCs; Supplementary Figure S3), indicating that emergence of large and fast EPSCs is not ascribed to adaptation to mean contrast increment (Dark BG vs. Static BG) but to alteration induced by the global jitter motion (Static BG vs. Jittered BG).

In contrast, inhibitory postsynaptic currents (IPSCs; Figure 3D) were not affected by the Jittered BG (amplitude, Jittered BG, $99.1 \pm 64.5$ pA; Dark BG, $102.6 \pm 66.9$ pA; kinetics, Jittered BG, $28.1 \pm 15.5$ ms, Dark BG $27.2 \pm 16.7 \mathrm{~ms}$, ps > 0.3; paired $t$-test, 5 Ft GCs; Figures 3E,F). Therefore, it is likely that the RF alterations under the Jittered BG condition were induced not by feedforward inhibitory synaptic inputs from amacrine cells (ACs) to Ft GCs but by excitatory synaptic inputs from bipolar cells (BCs) to Ft GCs.

\section{Alterations of the Spatial Distribution of Excitatory Inputs to Ft GCs by the Jittered BG}

To examine the spatial RF profile under the Jittered BG condition in detail, we mapped the distribution of EPSCs evoked by local light stimulation (Spatial EPSC map; Figure 4). A wholecell voltage-clamped Ft GC was stimulated by a small flash (Figure 4A; size, $100 \times 100 \mu \mathrm{m}$; duration, $50 \mathrm{~ms}$; interflash interval, $1 \mathrm{~s}$ ), which was presented randomly at various locations on the Dark BG (Figure 4B) or on the Jittered BG (Figure 4C). We found that the EPSC map under the Jittered BG condition was spatially expanded along the retinal horizontal axis (Figures 4C-E), corresponding to the horizontally elongated $\mathrm{RF}_{\text {JitteredBG }}$ (Figure 2D).

Interestingly, we found that the properties of evoked EPSCs were not homogeneous across the RF under the Jittered BG condition: small and slow inputs at the center region (Figure 4D [2] and Figure 4F; $17.8 \pm 9.87 \mathrm{pA}, 9.54 \pm 1.54 \mathrm{~ms}$ ); large and fast inputs at the horizontally-expanded region (Figure 4D [1] and Figure 4F; $43.7 \pm 19.39$ pA, $7.54 \pm 1.53 \mathrm{~ms}$ ). It is likely that the emergence of large and fast EPSCs mediates the horizontally elongated RF of Ft GCs under the Jittered BG condition.

\section{Contribution of GABAergic and Electrical Pathways to RF Elongation in Ft GCs}

In the goldfish retina both GABAergic inhibition from AC to Mb1 (ON) BC terminal (Tachibana and Kaneko, 1988) and electrical coupling through connexin $36(\mathrm{Cx} 36)$ between Mb1 BC dendrites contribute to global information processing (Arai et al., 2010; Tanaka and Tachibana, 2013; Matsumoto and Tachibana, 2017). It is possible that the horizontal RF elongation in Ft GCs may be attributable to deactivation of lateral inhibition mediated by ACs and/or activation of electrically coupled excitatory network (Bloomfield and Völgyi, 2009; Werblin, 2011). 
Using a multi-electrode array, we examined the effects of pharmacological blockers on the properties of $\mathrm{RF}_{\text {JitteredBG }}$ (Figure 5). Application of a GABA receptor blocker picrotoxin (PTX, $100 \mu \mathrm{M}$ ) increased the $\mathrm{RF}_{\text {DarkBG }}$ size (horizontal length, $142.7 \pm 12.4 \mu \mathrm{m}$ in Control, $162.3 \pm 18.1 \mu \mathrm{m}$ in PTX, $p=0.03$; vertical length, $133.5 \pm 13.9 \mu \mathrm{m}$ in Control, $158.6 \pm 14.8 \mu \mathrm{m}$ in PTX, $p=0.01$; Mann-Whitney $U$ (MWU) test, 7 Ft GCs; Figures $5 \mathrm{~B}, \mathrm{C})$. Even in the presence of PTX, the Jittered BG increased further the RF size $\left(\mathrm{RF}_{\mathrm{JitteredBG}}\right.$, horizontal length $194.5 \pm 17.9 \mu \mathrm{m}, p=0.007$; vertical length $208.2 \pm 27.3 \mu \mathrm{m}$, $p=0.0012$; MWU test, 7 Ft GCs, Figure 5C), although the spatial bias disappeared (Figure 5D). These results suggest that two different GABAergic mechanisms may mediate the surround inhibition (Werblin, 2011): the local inhibition which works under the dark BG condition, and the global inhibition which contributes to emphasizing the horizontal bias of the RF under the Jittered BG condition.

In the presence of gap junction blocker mefloquine (MFQ, $10 \mu \mathrm{M}$; Cruikshank et al., 2004), the Jittered BG did not increase the horizontal length of the $\mathrm{RF}_{\text {JitteredBG }}$ (horizontal length, Dark BG, $140.7 \pm 16.1 \mu \mathrm{m}$; Jittered BG, $132.7 \pm 14.8 \mu \mathrm{m}$, $p=0.32$; MWU test, 7 Ft GCs; Figures 5B,C), but decreased

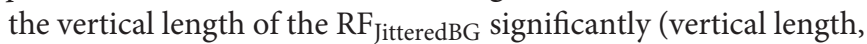
$130.3 \pm 15.9 \mu \mathrm{m}$ under the Dark BG, $107.3 \pm 8.3 \mu \mathrm{m}$ under the Jittered BG, $p=0.0175$; MWU test, 7 Ft GCs; Figure 5C). These results suggest that electrical coupling may mediate the spatial expansion of the $\mathrm{RF}_{\mathrm{JitteredBG}}$. Indeed, spatial bias index (see section "Method Details") indicates that the horizontal bias of the $\mathrm{RF}_{\text {JitteredBG }}$ was still maintained in the presence of MFQ (Figure 5D, green), whereas the horizontal bias was not observed in the presence of PTX (Figure 5D, red).

Therefore, the potential mechanism for the RF alterations may be the following: the Jittered BG expands the RF in all directions by electrical coupling, perhaps between Mb1 BCs (Arai et al., 2010); and the dorsal and ventral sides of the expanded RF are suppressed by activated GABAergic lateral inhibition (Tanaka and Tachibana, 2013), resulting in the horizontally elongated RF (Figure 5E). Lateral inhibition may be ascribed not to the feedforward input from AC to Ft GC but to the feedback input from AC to Mb1 BC terminal (Tanaka and Tachibana, 2013) because IPSCs in Ft GCs were not altered by the Jittered BG (Figures 3E,F).

\section{DISCUSSION}

In our previous study, we showed that firing properties of specific GCs in the goldfish retina were modulated by a rapid shift of a target following a period of jitter motion of a global randomdot background (Matsumoto and Tachibana, 2017). Here, we examined how global jitter motion alters the RF properties of Ft GCs and what mechanisms underlies the RF alteration. We found that global jitter motion induced the RF elongation along the retinal horizontal (caudal-rostral) axis (Figure 2) in Ft GCs (Figure 1). At the elongated region, EPSCs with large amplitude and fast kinetics were dominated (Figures 3, 4). Pharmacological experiments revealed that both GABAergic lateral inhibition and electrical coupling contributed to the horizontal RF elongation (Figure 5).

\section{Synaptic Modulation Mediated by Electrical and GABAergic Pathways}

Our results indicate that the RF alteration in Ft GCs is mediated by lateral interaction pathways: electrical and GABAergic pathways (Figure 5). In the goldfish retina, excitation of an Mb1 BC spreads to neighboring Mb1 BCs through gap junction between their dendrites (Arai et al., 2010). Such lateral spread of excitation could expand the RF. It is possible that the synchronous transient glutamate release from electrically coupled BCs may evoke EPSCs with large amplitude and fast kinetics. It is conceivable that Ft GCs may receive excitatory inputs from a population of BCs under the Dark BG ("conventional BCs" in Figure 5E), and large and fast excitatory inputs from another population of electrically coupled BCs which are additionally activated by the Jittered BG ("Electrically coupled BCs" in Figure 5E).

In the goldfish retina, global stimulation activates the electrically coupled Mb1 BC network, which in turn activates wide-field ACs and/or electrically coupled ACs (Tanaka and Tachibana, 2013). Blockade of GABA receptors under the Dark BG condition increased the size of RF in all directions (Figure 5C), indicating that the local GABAergic inhibition was not biased spatially (Figure 5E). However, blockade of GABA receptors under the Jittered BG condition impaired the horizontal bias (Figure 5D). These observations indicate that the global inhibitory mechanism could affect the electrically coupled $\mathrm{BCs}$ at the dorsal and vertical side of the expanded RF ("Global GABAergic inhibition," Figure 5E).

On the other hand, the horizontal bias remained even after blockade of electrical coupling (Figure 5D). This result indicates that the inputs from electrically coupled BCs may not be the unique resource to drive the global inhibitory mechanism. It is possible that the continuous jitter motion may prevent the BCs from adaptation, resulting in augmentation of the synaptic inputs to the wide-field ACs. Another possible resource may be the ACs electrically coupled through non-Cx36 (Mark et al., 1988; Völgyi et al., 2013), which are also activated by the Jittered BG. This mechanism could explain the observed horizontal bias in the presence of a gap junction blocker. Since AC circuits in the goldfish retina are not yet fully understood, further studies are needed to clarify the circuit mechanism.

\section{Diverse Features of Fixational Eye Movements}

In this study we used randomly jittering motion to mimic the retinal image during fixation, which was similar to the random walk used in the previous studies (Ölveczky et al., 2003; Baccus et al., 2008; Segal et al., 2015). The stimulus was summarized by oscillatory image motion with small amplitude, which approximates to an animal's tremor (e.g., archer fish, $12 \mu \mathrm{m}$ in mean amplitude, $5 \mathrm{~Hz}$ in frequency, Segev et al., 2007). Nevertheless, the features of fixational eye movements are more complicated in amplitude, speed, and frequency: drift, slow motion occurring together with tremor; microsaccedes, 
small but rapid and involuntary shifts of eyes (MartinezConde et al., 2004; Rucci and Poletti, 2015). Global jitter motion used in this study may be similar to tremor and drift in periodicity and slow frequency components $(0.5-8 \mathrm{~Hz}$; Supplementary Figure S2C). On the other hand, our stimulus did not include the small and rapid displacements corresponding to microsaccades. Furthermore, it is not yet clear how three components of fixational eye movements interact with one another and affect the retinal circuit. It is likely that that the RF alterations shown in this study may be a part of the effects induced by fixational eye movements. Further studies are required to dissect the relationship between the diverse features of fixational eye movements and the retinal information processing.

\section{Functional Relevance of RF Elongation}

Goldfish makes horizontally biased saccades spontaneously (Salas et al., 1997). Thus, the horizontal elongation of RF in Ft GCs during global jitter motion seems to be advantageous to responding quickly to a target in a succeeding rapid gaze shift (saccade). Indeed, we have shown that horizontal, but not vertical, rapid shift of a target following jitter motion evoked firing in Ft GCs before arrival of the target to the RF estimated under the Dark BG condition (Matsumoto and Tachibana, 2017). In some visual neurons in the brain, their RFs spatially shift or expand toward the future position of eyes before saccade initiation (frontal eye field; Umeno and Goldberg, 1997, prefrontal cortex; Zirnsak et al., 2014). These dynamic RF changes in visual neurons may contribute to fast and efficient processing of visual information during repetitive sequence of eye movements (Hosoya et al., 2005; Gollisch and Meister, 2008; Wurtz, 2008; Zirnsak et al., 2014). It is possible that, in natural environment, the RF profiles of specific GCs are tuned to global image motion induced by eye movements to facilitate processing in the visual system.

\section{REFERENCES}

Arai, I., Tanaka, M., and Tachibana, M. (2010). Active role of electrical coupled bipolar cell network in the adult retina. J. Neurosci. 30, 9260-9270. doi: 10.1523/ JNEUROSCI.1590-10.2010

Baccus, S. A., Ölveczky, B. P., Manu, M., and Meister, M. (2008). A retinal circuit that computes object motion. J. Neurosci. 28, 6807-6817. doi: 10.1523/ JNEUROSCI.4206-07.2008

Baden, T., Berens, P., Franke, K., Román Rosón, M., Bethge, M., and Euler, T. (2016). The functional diversity of retinal ganglion cells in the mouse. Nature 529, 345-350. doi: 10.1038/nature16468

Bair, W., and O'Keefe, L. P. (1998). the influence of fixational eye movements on the response of neurons in area $\mathrm{mt}$ of the macaque. Vis. Neurosci. 5, 779-786. doi: $10.1017 / \mathrm{s} 0952523898154160$

Bloomfield, S., and Völgyi, B. (2009). The diverse functional roles and regulation of neuronal gap junctions in the retina. Nat. Rev. Neurosci. 10, 495-506. doi: $10.1038 / \mathrm{nrn} 2636$

Brainard, D. (1997). The psychophysics tool box. Spat. Vis. 10, 443-446.

Cruikshank, S., Hopperstad, M., Younger, M., Connors, B., Spray, D., and Srinivas, M. (2004). Potent block of Cx36 and Cx50 gap junction channels by mefloquine. Proc. Natl. Acad. Sci. U.S.A. 101, 12364-12369. doi: 10.1073/pnas.040204 4101

DeVries, S. H., and Baylor, D. A. (1997). Mosaic arrangement of ganglion cell receptive fields in rabbit retina. J. Neurophysiol. 78, 2048-2060. doi: 10.1152/ jn.1997.78.4.2048

\section{DATA AVAILABILITY}

The data and codes that used in this research are available at the corresponding author, MT (mstchbn@fc.ritsumei.ac.jp) upon reasonable requests.

\section{ETHICS STATEMENT}

The animal study was reviewed and approved by Committee of Animal Experiments in The University of Tokyo.

\section{AUTHOR CONTRIBUTIONS}

Both authors designed the study, performed all experiments, and wrote the manuscript. AM performed the data analysis.

\section{FUNDING}

This work was supported by Core Research for Evolutional Science and Technology, Japan Science and Technology Agency (CREST, JST) and JST KAKENHI Grant Number JP19K06915 to MT and by Grants-in-Aid for Scientific Research, Japan Society for the Promotion of Science (JSPS) Fellows to AM.

\section{SUPPLEMENTARY MATERIAL}

The Supplementary Material for this article can be found online at: https://www.frontiersin.org/articles/10.3389/fnins. 2019.00979/full\#supplementary-material

Easter, S., Pamela, R., and Heckenlively, D. (1974). Horizontal compensatory eye movements in goldfish (Carassius auratus). J. Comp. Physiol. 92, 23-35. doi: 10.1007/bf00696524

Gollisch, T., and Meister, M. (2008). Rapid neural coding in the retina with relative spike latencies. Science 319, 1108-1111. doi: 10.1126/science. 1149639

Gouwens, N. W., Sorensen, S. A., Berg, J., Lee, C., Jarsky, T., Ting, J., et al. (2019). Classification of electrophysiological and morphological neuron types in the mouse visual cortex. Nat. Neurosci. 22, 1182-1195. doi: 10.1038/s41593-0190417-0

Greschner, M., Bongard, M., Rujan, P., and Ammermüller, J. (2002). Retinal ganglion cell synchronization by fixational eye movements improves feature estimation. Nat. Neurosci. 5, 341-347. doi: 10.1038/nn821

Hilgen, G., Pirmoradian, S., Pamplona, D., Komprobst, P., Cessac, B., Hennig, M. H., et al. (2017). Pan-retinal characterization of light responses from ganglion cells in the developing mouse retina. Sci. Rep. 7:42330.

Hosoya, T., Baccus, S. A., and Meister, M. (2005). Dynamic predictive coding by the retina. Nature 436, 71-77. doi: 10.1038/nature03689

Keesey, U. T. (1960). Effects of involuntary eye movements on visual acuity. J. Opt. Soc. Am. 50:769. doi: 10.1364/josa.50.000769

Krishnamoorthy, V., Weick, M., and Gollisch, T. (2017). Sensitivity to image recurrence across eye-movement-like image transitions through local serial inhibition in the retina. eLife 6:e22431. doi: 10.7554/eLife.22431

Land, M. F. (2009). Vision, eye movements, and natural behavior. Vis. Neurosci. 26, 51-62. doi: 10.1017/S0952523808080899 
Lewicki, M. S. (1998). A review of methods for spike sorting: the detection and classification of neural action potentials. Network 9, R53-R78.

Macy, A., and Easter, S. (1981). Growth-related changes in the size of receptive field centers of retinal ganglion cells in goldfish. Vis. Res. 21, 1497-1504. doi: 10.1016/0042-6989(81)90221-2

Mark, R. E., Liu, W. L., and Muller, J. E. (1988). Gap junctions in the inner plexiform layer of the goldfish retina. Vis. Res. 28, 9-24. doi: 10.1016/00426989(88) $90003-x$

Martínez, J. J., Rahsepar, B., and White, J. A. (2017). Anatomical and electrophysiological clustering of superficial medial entorhinal cortex interneurons. eNeuro 4:ENEURO.0263-16.2017. doi: 10.1523/ENEURO. 0263-16.2017

Martinez-Conde, S., Macknik, S., and Hubel, D. (2004). The role of fixational eye movements in visual perception. Nat. Rev. Neurosci. 5, 229-240. doi: 10.1038/ nrn1348

Martinez-Conde, S., Macknik, S. L., and Hubel, D. H. (2000). Microsaccadic eye movements and firing of single cells in the striate cortex of macaque monkeys. Nat. Neurosci. 3, 251-258. doi: 10.1038/72961

Matsumoto, A., and Tachibana, M. (2017). Rapid and coordinated processing of global motion images by local clusters of retinal ganglion cells. Proc. JPN. Acad. Ser. B Phys. Biol. Sci. 93, 234-249. doi: 10.2183/pjab.93.015

Meister, M., Pine, J., and Baylor, D. A. (1994). Multi-neuronal signals from the retina: acquisition and analysis. J. Neurosci. Methods 51, 95-106. doi: 10.1016/ 0165-0270(94)90030-2

Mensh, B., Aksay, E., Lee, D., Seung, H., and Tank, D. (2004). Spontaneous eye movements in goldfish: oculomotor integrator performance, plasticity, and dependence on visual feedback. Vis. Res. 44, 711-726. doi: 10.1016/j.visres.2003. 10.015

Murakami, I. (2006). Fixational eye movements and motion perception. Prog. Brain Res. 154, 193-209. doi: 10.1016/s0079-6123(06)54010-5

Noda, H., and Adey, W. R. (1974). Retinal ganglion cells of the cat transfer information on saccadic eye movement and quick target motion. Brain Res. 70, 340-345. doi: 10.1016/0006-8993(74)90323-0

Ölveczky, B. P., Baccus, S. A., and Meister, M. (2003). Segregation of object and background motion in the retina. Nature 423, 401-408. doi: 10.1038/ nature 01652

Pelli, D. (1997). The videotoolbox software for visual psychophysics: transforming numbers into movies. Spat. Vis. 10, 437-442. doi: 10.1163/156856897x00366

Rey, H. G., Pedreira, C., and Quian Quiroga, R. (2015). Past, present and future of spike sorting techniques. Brain Res. Bull. 119, 106-117. doi: 10.1016/j. brainresbull.2015.04.007

Roska, B., and Werblin, F. (2003). Rapid global shifts in natural scenes block spiking in specific ganglion cells types. Nat. Neurosci. 6, 600-608. doi: 10.1038/ nn1061

Rousseeuw, P. J. (1987). Silhouettes: a graphical aid to the interpretation and validation of cluster anslysis. J. Comput. Appl. Math. 20, 53-65. doi: 10.1016/ 0377-0427(87)90125-7

Rucci, M., Iovin, R., Poletti, M., and Santini, F. (2007). Miniature eye movements enhance fine spatial detail. Nature 447, 852-855. doi: 10.1038/nature05866

Rucci, M., and Poletti, M. (2015). Control and functions of fixational eye movements. Annu. Rev. Vis. Sci. 1, 499-518. doi: 10.1146/annurev-vision082114-035742

Salas, C., Herrero, L., Rodriguez, F., and Torres, B. (1997). Tectal codification of eye movements in goldfish studied by electrical microstimulation. Neuroscience 78, 271-288. doi: 10.1016/s0306-4522(97)83048-5
Segal, I. Y., Giladi, C., Gedalin, M., Rucci, M., Ben-Tov, M., Kushinsky, Y., et al. (2015). Decorrelation of retinal response to natural scenes by fixational eye movements. Proc. Natl. Acad. Sci. U.S.A. 112, 3110-3115. doi: 10.1073/pnas. 1412059112

Segev, R., Schneidman, E., Goodhouse, J., and Berry, M. J. (2007). Role of eye movements in the retinal code for a size discrimination task. J. Neurophyiol. 98, 1380-1391. doi: 10.1152/jn.00395.2007

Tachibana, M., and Kaneko, A. (1988). Retinal bipolar cells receive negative feedback input from GABAergic amacrine cells. Vis. Neurosci. 1, 297-305. doi: $10.1017 /$ s0952523800001954

Tanaka, M., and Tachibana, M. (2013). Independent control of reciprocal and lateral inhibition at the axon terminal of retinal bipolar cells. J. Physiol. 591, 3833-3851. doi: 10.1113/jphysiol.2013.253179

Tien, N. W., Pearson, J. T., Heller, C. R., Demas, J., and Kerschensteiner, D. (2015). Genetically identified suppressed-by-contrast retinal ganglion cells reliably signal self-generated visual stimuli. J. Neurosci. 35, 10815-10820. doi: 10.1523/ JNEUROSCI.1521-15.2015

Tulunay-Keesey, U., and Jones, R. M. (1976). The effects of micromovements of the eye and exposure duration on contrast sensitivity. Vis. Res. 16, 481-488. doi: 10.1016/0042-6989(76)90026-2

Umeno, M. M., and Goldberg, M. E. (1997). Spatial processing in the monkey frontal eye field. I. predictive visual responses. J. Neurophysiol. 78, 1373-1383. doi: 10.1152/jn.1997.78.3.1373

Völgyi, B., Kovács-Oller, T., Atlasz, T., Wilhelm, M., and Gábriel, R. (2013). Gap junctional coupling in the vertebrate retina: variations on the theme? Prog. Retin. Eye Res. 34, 1-18. doi: 10.1016/j.preteyeres.2012. 12.002

Werblin, F. (2011). The retinal hypercircuit: a repeating synaptic interactive motif underlying visual function. J. Physiol. 589, 3691-3702. doi: 10.1113/jphysiol. 2011.210617

Wurtz, R. (2008). Neuronal mechanisms of visual stability. Vis. Res. 48, 2070-2089. doi: 10.1016/j.visres.2008.03.021

Yarbus, A. L. (1967). Eye Movements and Vision. New York, NY: Plenum.

Yuan, C., and Yang, H. (2019). Research on K-value selection method of K-means clustering algorithm. J. Multidiscip. Sci. 2, 226-235. doi: 10.3390/j20 20016

Zhang, P. M., Wu, J. Y., Zhou, Y., Liang, P. J., and Yuan, J. Q. (2004). Spike sorting based on automatic template reconstruction with a partial solution to the overlapping problem. J. Neurosci. Methods 135, 55-65. doi: 10.1016/j. jneumeth.2003.12.001

Zirnsak, M., Steinmetz, N. A., Noudoost, B., Xu, K. Z., and Moore, T. (2014). Visual space is compressed in prefrontal cortex before eye movements. Nature 507, 504-507. doi: 10.1038/nature 13149

Conflict of Interest Statement: The authors declare that the research was conducted in the absence of any commercial or financial relationships that could be construed as a potential conflict of interest.

Copyright (c) 2019 Matsumoto and Tachibana. This is an open-access article distributed under the terms of the Creative Commons Attribution License (CC BY). The use, distribution or reproduction in other forums is permitted, provided the original author(s) and the copyright owner(s) are credited and that the original publication in this journal is cited, in accordance with accepted academic practice. No use, distribution or reproduction is permitted which does not comply with these terms. 